\title{
Design and Simulation of an Optical Gigabit Ethernet Network
}

\author{
Salah A. Jaro Alabady \\ College of Engineering \\ Computer Engineering. Dept. \\ salah_alabady@yahoo.com
}

\author{
Omar Ahmed Yousif \\ College of Engineering \\ Computer Engineering. Dept. \\ omar_hachum@yahoo.com
}

\begin{abstract}
This paper deals with the design and simulation of an optical gigabit Ethernet using OPTSIM 3.6 packet software. The main aim of the proposed design is to build a MAN optical network using one-gigabit Ethernet technique, and what are the necessary requirements to build these networks. As a case study, all states center are connected as Star - Bus topology using layer2 and layer3 optical switches. In addition, in this paper one-gigabit optical transmitter and receiver are designed to work as a node in the network topology. Further more, the benefits of using $L$ - Band wavelength for transmission take in consider the linear and non-linear effects on fiber optic is presented.
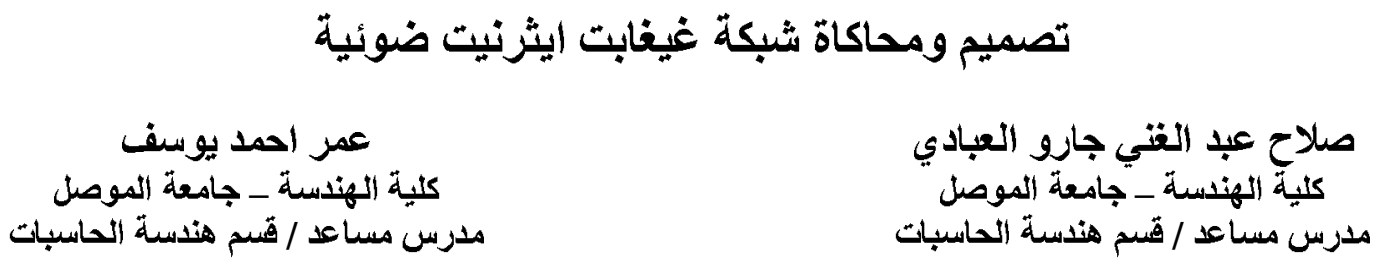

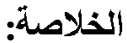

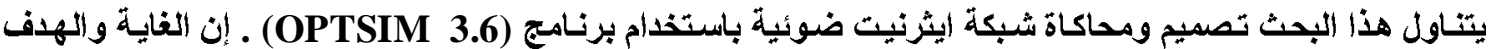

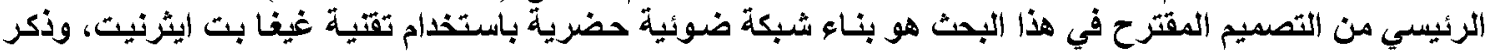

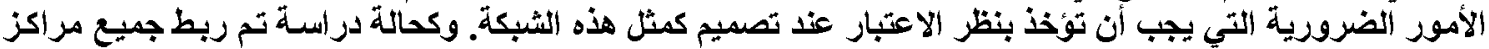

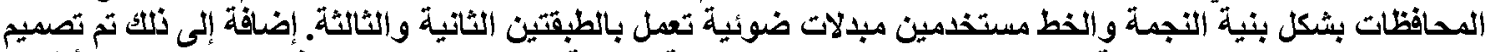

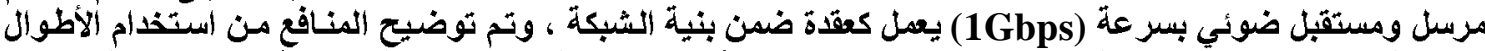
الموجية التي تقع ضمن الحزمة (L) اخذين بنظر الاعتبار التأثيرات الخطية وغير الخطية على الألياف الضوئية.
\end{abstract}

Keyword: - Optical communications, Optical networks, Optical gigabit Ethernet 


\section{1- Introduction}

Increasingly networks are being converted from copper to optical fiber. As a result, network design procedures need to be adjusted to address the specific requirements of fiber optic cable as a communications transport media. The use and demand for optical fiber had grown tremendously and optical-fiber applications are numerous. Telecommunication applications are widespread, ranging from global networks to desktop computers. These involve the transmission of voice, data, or video over distances range from less than a meter to hundreds of kilometers, by using one of a few standard fiber optic designs instead of several copper cable designs.

All networks involve the same basic principles: information can be sent to, shared with, passed on, or bypassed within a number of computer stations (nodes) and a master computer (server). Network applications include LANs, MANs, WANs, SANs, intrabuilding and interbuilding communications, broadcast distribution, intelligent transportation systems (ITS), telecommunications, etc. In addition to its advantages (i.e. bandwidth, durability, ease of installation, immunity to EMI/RFI and harsh environmental conditions, long-term economies, etc.), optical fiber better accommodates today's increasingly complex network architectures than copper alternatives [1-3].

Gigabit Ethernet solutions have become a necessity with the accelerating growth of LAN and MAN traffic. The fundamental designing objectives in an optical network concern the optimizations regarding the following two metrics: The first one is to minimize the network total cost. The second one is to maintain an acceptable quality of service (QoS), and good performance. This paper provides, detailed general guidance to design optical network using gigabit Ethernet technique at longer wavelength, and give a case study to connect all universities in IRAQ.

\section{2 - Overview of Optical Gigabit Ethernet}

Optical gigabit Ethernet solutions have become a necessity with the accelerating growth of LAN traffic, pushing network administrators to look for higher speed network technologies to meet the demand for more bandwidth $[2,3]$.

While most copper systems will support Gigabit Ethernet, fiber optics provides a much higher degree of flexibly and future bandwidth/speed expansion compared with its copper counterparts. Generally, copper will support Gigabit and multi-gigabit transmission rates, but only for very short distances. Copper is affected by EMI (electromagnetic interference) and RFI (radio frequency interference). Fiber optics will support Gigabit and multi-gigabit transmission for both short and long distances, with immunity to EMI and RFI, making fiber more suitable solution for a number of applications.

Depending on the fiber type and core size, Gigabit Ethernet applications supported by fiber optics are now transmitting signal reliably at $10 \mathrm{Gbps}$, up to $80 \mathrm{~km}$ using single mode systems, and well over that for Gigabit and multi-gigabit transmission rates. While fiber optics provide some clear advantages over copper legacy technology, most systems using fiber today also use copper at the end user point, creating a hybrid system for data transmission. The advantages in utilizing a hybrid system exists by leveraging the bandwidth and EMI/RFI advantages of fiber for the longer length and main distribution lines of the network, while using copper for the very short desktop and non-backbone connectivity, allowing a very easily routable and inexpensive connectivity solution without implementing media converters $[3,4]$. 


\section{3 - System Design and Simulation of Optical Networks}

The two key parameters, which affect the maximum cable length or maximum possible repeater spacing and the maximum possible bit rate, are attenuation and dispersion [5]. Signal attenuation in fiber is expressed in $\mathrm{dB} / \mathrm{km}$. The $\mathrm{dB}$ (Decibel ) is used for comparing two power levels and is defined as the ratio of the output power (PO) to the input power (PI). The power budget for a data network is important because we have to ensure that we have enough power in the source to reach the farthest station without saturating the nearest station. (This is the dynamic range of the source.)

The following table 1 shows the typical Attenuation Loss for each component [6].

Table (1) : Typical Attenuation Loss

\begin{tabular}{||l|l||}
\hline COMPONENT & LOSS VALUE \\
\hline Adapter & $3 \mathrm{~dB}$ \\
\hline Splice Joint & $0.1 \mathrm{~dB}$ \\
\hline Single mode Fiber Cable @ 1580nm & $0.2 \mathrm{~dB} / \mathrm{km}$ \\
\hline Safe margin & $3 \mathrm{~dB}$ \\
\hline
\end{tabular}

\section{1 - Fiber Span Analysis}

Span analysis is the calculation and verification of a fiber-optic system's operating characteristics. This encompasses items such as fiber routing, electronics, wavelengths, fiber type, and circuit length. Attenuation and nonlinear considerations are the key parameters for loss-budget analysis. Before implementing or designing a fiber-optic system, a span analysis is recommended to make sure that the system must work over the proposed link. Both the passive and active components of the system have to be included in the loss-budget calculation. Passive loss is made up of fiber loss, connector loss, splice loss, and losses involved with couplers or splitters in the link. Active components are system gain, transmitter power, receiver sensitivity, and dynamic range $[2,4]$.

The overall span loss, or link budget as it is sometimes called, can be determined by using an optical meter to measure true loss or by computing the loss of the system components. The latter method considers the loss associated with span components, such as connectors, splices, patch panels, jumpers, and the optical safety margin. The safety margin sets aside $3 \mathrm{~dB}$ to compensate for component aging and repair work in event of fiber cut. Adding all of these factors to make sure that their total sum is within the maximum attenuation figure, in order to ensure that the system will operate satisfactorily. Allowances must also be made for the type of splice, equipment, and the environment (including temperature variations).

Nonlinear effects occur at high bit rates and power levels. These effects must be mitigated using compensators, and a suitable budget allocation must be made during calculations. Nonlinear effects of fiber became apparent with specialized applications such as undersea installations. Some of these effects are important to know when designing fiber optics systems, include: Stimulated Brillouin Scattering (SBS), Stimulated Raman Scattering (SRS), Four Wave Mixing (FWM), Self-Phase Modulation (SPM), Cross-Phase Modulation (XPM), and inter-modulation (mixing). Non-linear effects limit the amount of data that can be transmitted on a single optic fiber. System designers must be aware of these limitations and the steps that can be taken to minimize the detrimental effects of fiber non-linearities.

Linear effects include Attenuation and Dispersion. A fiber with a lower attenuation will allow more power to reach its receiver than a fiber with higher attenuation. Figure (1) shows 
the BER versus wavelength, this figure demonstrates that the longest wavelength $(1580 \mathrm{~nm}$ in this case) is the best for transmitted because the attenuation in this wavelength is lower than another wavelength. Also the dispersion equal zero at this wavelength as shown in figure (2). We can calculate the bit error rate (BER) and plotted the wavelength versus BER using equation (1)

$B E R=\frac{1}{2} \operatorname{erfc}\left(\frac{Q}{\sqrt{2}}\right)$

Where erfc ( ) is the complementary error function

The Q-factor for a Gaussian shape pulse is given by:

$$
Q=\frac{I_{1}-I_{0}}{\sigma_{H}+\sigma_{L}}
$$

These currents, can be calculated using the idea of counting the number of electrons incident Where the $I_{1} \& I_{0}$ are the currents obtained for 'one' and 'zero' logic's respectively. at the photo detector when a logic 'one' or logic 'zero' is received.

$$
Q=\frac{m_{1}-m_{0}}{\sqrt{m_{1}}+\sqrt{m_{0}}}=\sqrt{m_{1}}-\sqrt{m_{0}}
$$

Where:

$$
\begin{gathered}
m_{0}=m_{b 0}+m_{d} \text {, the average number of electrons representing the 'zero' symbol } \\
m_{1}=m_{b 1}+m_{d} \text {, the average number of electrons representing the 'one' symbol } \\
m_{d}=\frac{{ }_{d} T}{q} \text {, the average number of electrons correspond the dark current } i_{d} \text { during the } \\
m_{b 0}=\frac{\eta \lambda}{h c} p_{0} T \\
m_{b 1}=\frac{\eta \lambda}{h c} p_{1} T
\end{gathered}
$$

where:

$\eta$ efficiency of photo detector

$\lambda$ wavelength of photo transmitter

h Blank constant $\left(6.625 * 10^{-34}\right.$ J.S $)$

c light speed $\left(3 * 10^{+8} \mathrm{~m} / \mathrm{s}\right)$

q electron charge $\left(1.602 * 10^{-19} \mathrm{C}\right)$

$i_{d}$ dark current

$\mathrm{p}_{\mathrm{o}}$ optical power representing the 'zero' symbol

$\mathrm{p}_{1}$ optical power representing the 'one' symbol

Dispersion will have an impact on the proposed system transmission because it will increase the pulse broadening of the transmitted data, which leads to pulse distortion. 
Chromatic dispersion for CorningLEAF_submarine fiber can is characterized by.[9]

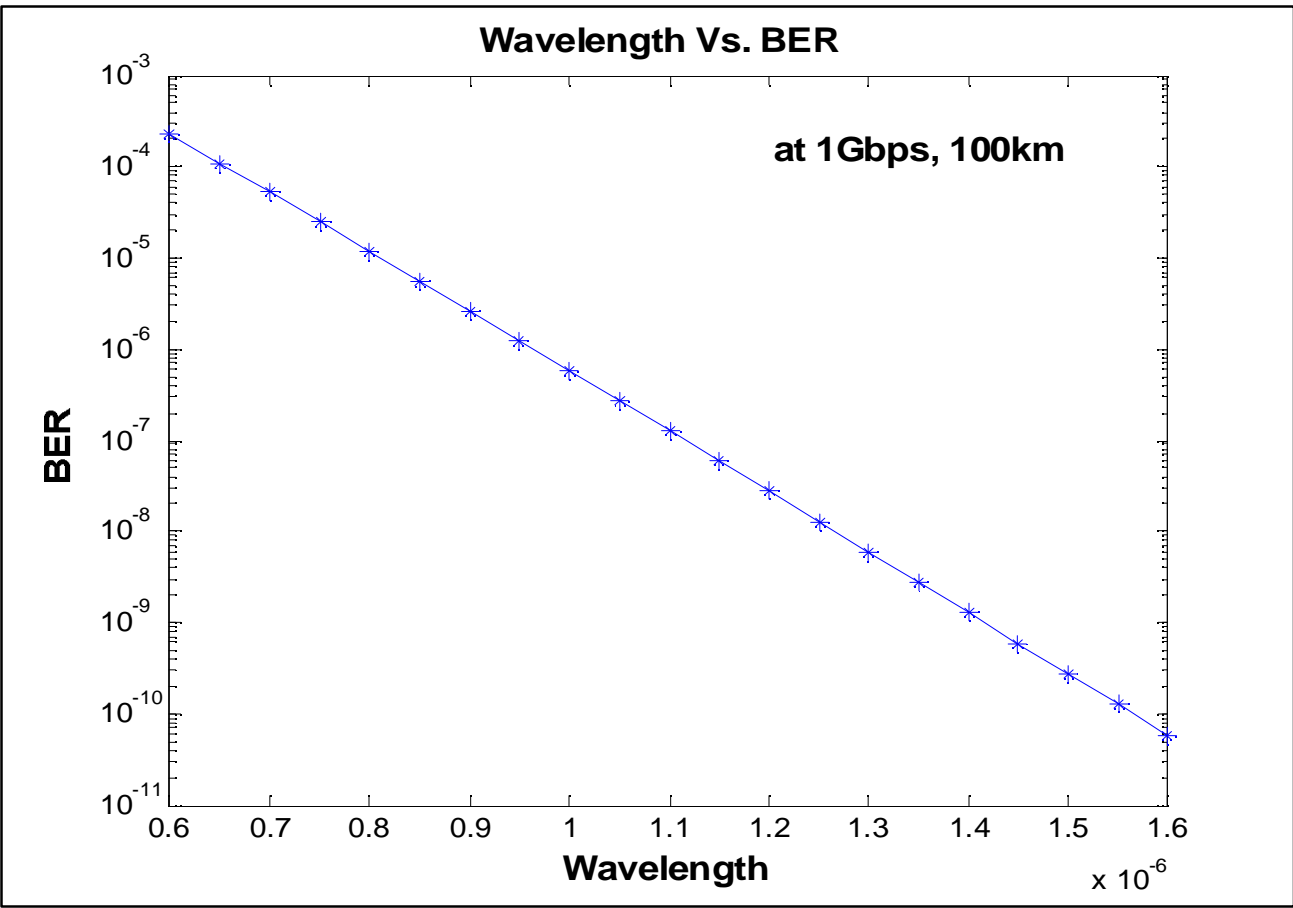

Fiqure (1): Wavelenqth versus BER

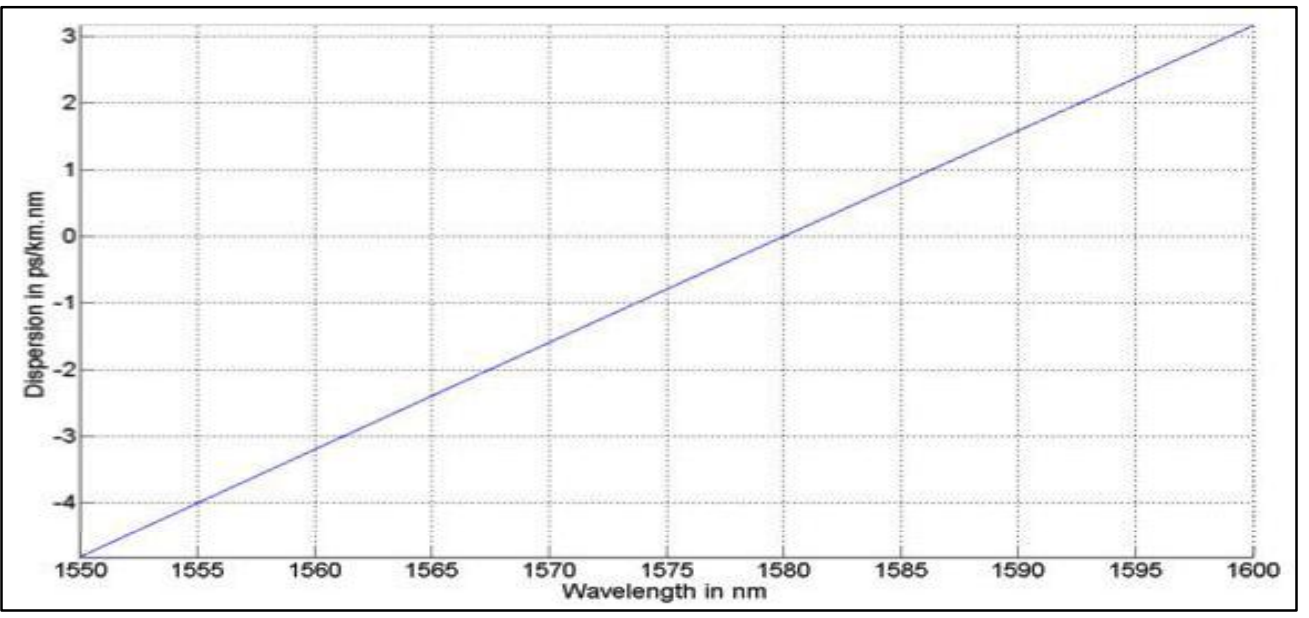

Figure (2): Dispersion of Corning LEAF_Submarine Fiber

$D(\lambda) \approx \lambda_{0} \cdot S_{0} \cdot \ln \left[\frac{\lambda}{\lambda_{0}}\right]$

Where:

$\mathrm{S}_{0}$ is the zero-dispersion wavelength $=0.11 \mathrm{ps} / \mathrm{km} \cdot \mathrm{nm}^{2}$ value .

$\mathrm{A}_{\mathrm{eff}}=\mid \mathbf{1} \perp \mathrm{m}^{2}$ for CorningLEAF_submarine fiber.

The effective length $\left(L_{\text {eff }}\right)$ is characterized by [10] 


$$
L_{e f f}=\frac{1}{\alpha}\left(1-e^{-\alpha L}\right)
$$

Where:

$\mathrm{L}:$ is the actual fiber length,

$\alpha:$ is the fiber attenuation constant in $1 / \mathrm{km}$ characterized by the equation [10]

$\alpha=\frac{A}{10 \log e}$

Where:

$A=0.2:$ is maximum fiber attenuation in $\mathrm{dB} / \mathrm{km}$ at the $1580 \mathrm{~nm}$.

Dispersion for CorningLEAF_submarine fiber versus transmitted wavelength can be plotted using equation (4) as shown in Figures (2). The analysis in Figure (2) shows the dispersion of Corning LEAF_submarine fiber, in the best case, will required to send data at wavelength $1580 \mathrm{~nm}$.

\section{2 - Stimulated Raman Scattering (SRS)}

When light propagates through a medium, the photons interact with silica molecules during propagation. The photons also interact with themselves and cause scattering effects, such as stimulated Raman scattering (SRS), in the forward and reverse directions of propagation along the fiber. This results in a sporadic distribution of energy in a random direction. SRS refers to shorter wavelengths pumping up the amplitude of longer wavelengths, which results in the longer wavelengths suppressing signals from the shorter wavelengths. One way to mitigate the effects of SRS is done by decreasing the input power [2]. In SRS, a short wavelength wave called Stoke's wave is generated due to the scattering of energy. This wave amplifies the higher wavelengths. The gain obtained by using such waveforms is considered in the Raman amplification basis. The Raman gain can extend most of the operating bands (C- and L-bands) for WDM networks. SRS is pronounced at high bit rates and high power levels. The margin design requirement to account for SRS/SBS is $0.5 \mathrm{~dB}$ $[2,3]$.

The $3 \mathrm{~dB}$ power threshold for SRS can be calculated by the following formula [10].

$$
P_{t h}(S R S)=16 A_{e f f} / g_{R} L_{e f f}
$$

Where: $S R S$ gain $g_{R}=1 \times 10^{-13} \mathrm{~m} / \mathrm{W}$.

$\mathrm{A}_{\mathrm{eff}}=\square 1 \square \mathrm{m}^{2}$ for CorningLEAF_submarine fiber.

$\mathrm{L}_{\text {eff }}$ can be calculated using equation (5)

The SRS power threshold verse fiber length can be plotted using equation (7) as shown in Figures (3). The SRS threshold power for the farthest distance between two cites (fiber length $250 \mathrm{~km})$ The $\mathrm{P}_{\mathrm{th}}(\mathrm{SRS})=27.1 \mathrm{dBm}(523 \mathrm{~mW})$ Therefore SRS will not be a concern for the proposed system. 


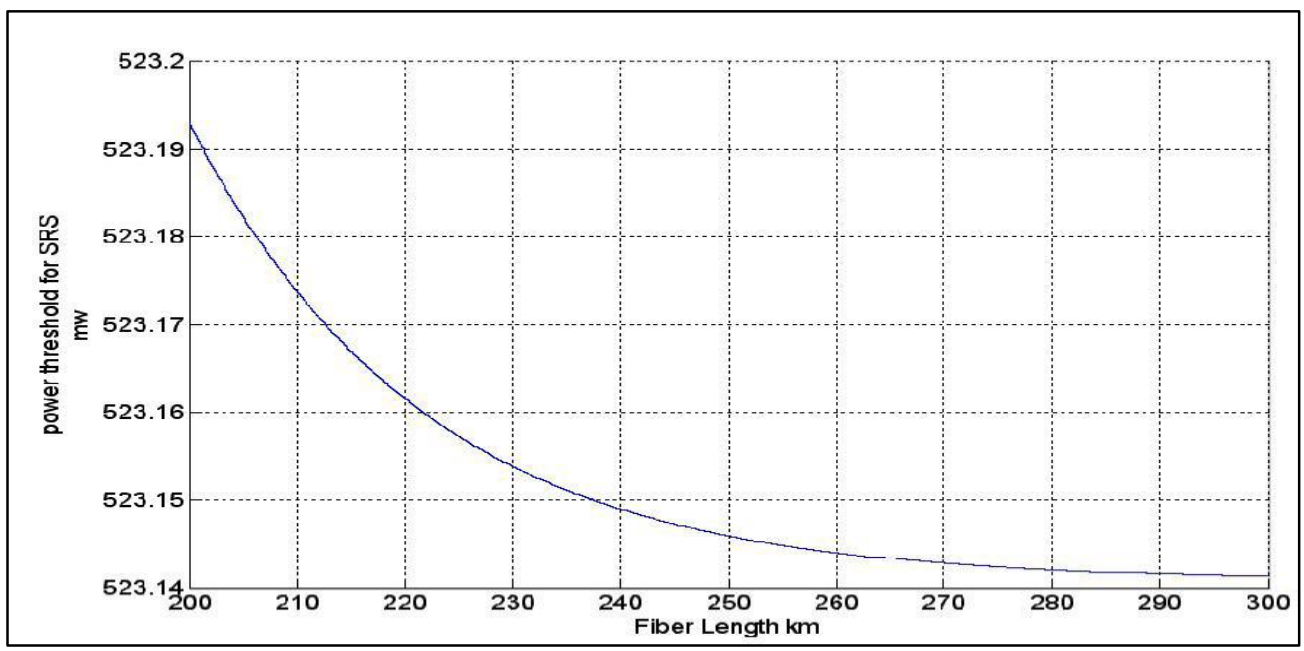

Figure (3): Stimulated Raman Scattering (SRS) power threshold versus fiber length

\section{3 - Stimulated Brillouin Scattering (SBS)}

Stimulated Brillouin scattering (SBS) is due to the acoustic properties of photon interaction with the medium [2]. Stimulated Brillouin scattering (SBS) is a nonlinear process that can occur in optical fibers at input power levels much lower than those needed for stimulated Raman scattering (SRS). It manifests through the generation of a backward- propagating Stokes wave that carries most of the input energy, once the Brillouin threshold is reached. Stimulated Brillouin scattering is typically harmful for optical communication systems. At the same time, it can be useful for making fiber-based Brillouin lasers and amplifiers [4]. It is similar to SRS in as much as it manifests through the generation of a Stokes wave whose frequency is downshifted from that of the incident light by an amount set by the nonlinear medium. However, major differences exist between SBS and SRS. For example, the Stokes wave propagates backward when SBS occurs in a single-mode optical fiber, in contrast to SRS that can occur in both directions. The Stokes shift $(\sim 10 \mathrm{GHz})$ is smaller by three orders of magnitude for SBS compared with that of SRS. The threshold pump power for SBS depends on the spectral width associated with the pump wave. It can be as low as $1 \mathrm{~mW}$ for a continuous-wave $(\mathrm{CW})$ pump or when the pumping is in the form of relatively wide pump pulses (width $>1 \mu \mathrm{s}$ ). In contrast, SBS nearly ceases to occur for short pump pulses (width $<10 \mathrm{~ns}$ ). All of these differences stem from single fundamental change acoustical phonons participate in SBS whereas optical phonons are involved in the case of SRS [4].

Rayleigh scattering, a major source of fiber losses, is an example of elastic scattering in which the frequency of scattered light remains unchanged. In contrast, the frequency is shifted downward during inelastic scattering. Raman and Brillouin processes provide two examples of inelastic scattering.

To calculate the $3 \mathrm{~dB}$ threshold power for SBS, the effective area (Aeff) and effective length $\left(\mathrm{L}_{\text {eff }}\right)$ must be known. The effective area $\left(\mathrm{A}_{\text {eff }}\right)$ can often be found in the manufacturer's data sheet.

The SBS 3dB power threshold follows the equation [10]. 


$$
P_{t h}(S B S) \approx 21 A_{e f f} / g_{B} L_{e f f}
$$

where :

SBS gain $\mathrm{g}_{\mathrm{B}}=5 \times 10^{-11} \mathrm{~m} / \mathrm{W}$

$\mathrm{A}_{\text {eff }}=\left\lceil 1 \sqcap \mathrm{m}^{2}\right.$ for CorningLEAF_submarine fiber.

$\mathrm{L}_{\text {eff }}$ can be calculated using equation (5).

The SBS power threshold versus fiber length can be plotted using equation (8) as shown in Figures (4). The SBS threshold power for the farthest distance between two cites (fiber length $250 \mathrm{~km}) \mathrm{P}_{\mathrm{th}}(\mathrm{SBS})=1.3 \mathrm{dBm}(1.3 \mathrm{~mW})$. This means we can assume the scattered power due to SBS will occur in the proposed system above $\mathrm{P}_{\mathrm{th}}(\mathrm{SBS})=1.3 \mathrm{dBm}$, we should limit launch power to less than $1.3 \mathrm{dBm}$.

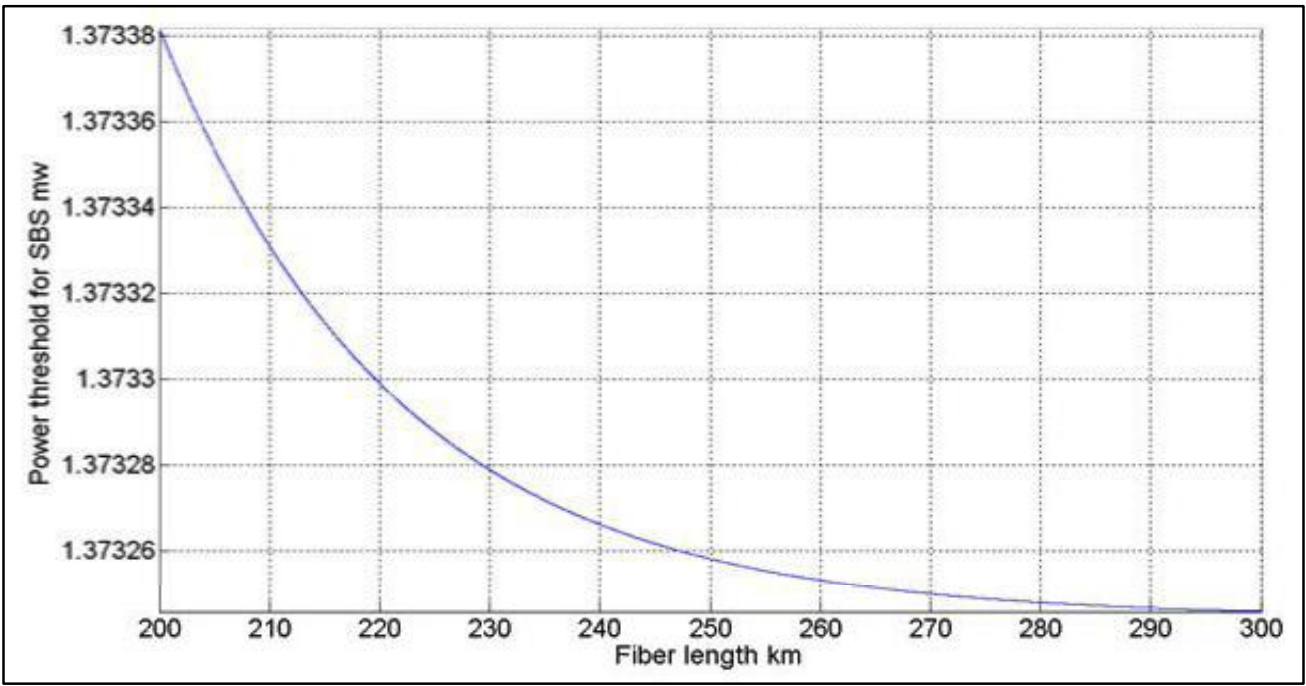

Figure (4): Stimulated Brillouin Scattering(SBS) power threshold

versus fiber length

\section{4 - Self-phase Modulation}

Phase modulation of an optical signal by itself is known as self-phase modulation (SPM). SPM is primarily due to the self-modulation of the pulses [11]. Generally, SPM occurs in single-wavelength systems. At high bit rates, however, SPM tends to cancel dispersion. SPM increases with high signal power levels. In fiber plant design, a strong input signal helps overcome linear attenuation and dispersion losses. However, consideration must be given to receiver saturation and to nonlinear effects such as SPM, which occurs with high signal levels. SPM results in phase shift and a nonlinear pulse spread. As the pulses spread, they tend to overlap and are no longer distinguishable by the receiver. The NRZ encoded in optical transmitter used in system suggested therefore , SPM phase shift in a NRZ digital system becomes significant when phase $\operatorname{shift}(\approx \pi / 2)[4]$.

Before the phase shift of the pulse can be calculated, the nonlinear propagation coefficient must be known. The nonlinear propagation coefficient is estimated by [10] 


$$
\gamma=2 \pi n / \lambda A_{e f f}
$$

Where:

The nonlinear index coefficient $\mathrm{n}=2.5 \times 10^{-20} \mathrm{~m}^{2} / \mathrm{W}$,

$\lambda=1580 \mathrm{~nm}$,

$\mathrm{A}_{\mathrm{eff}}=71 \mu \mathrm{m}^{2}$ for CorningLEAF_submarine fiber.

The $3 \mathrm{~dB}$ power threshold for SRS can be calculated by the following formula [10].

$$
\Phi(S P M)=\gamma P_{i n} L_{e f f}
$$

Where:

$\mathrm{L}_{\mathrm{eff}}$ can be calculated using equation (4) when the real fiber length $=250 \mathrm{~km}$.

Aeff $=71 \mathrm{~mm} 2$ for CorningLEAF_submarine fiber.

$\square=2.6 \times 10^{-3} / \mathrm{Wm}$ for MetroCor.

According to Figure (5) Self-phase Modulation in CorningLEAF_submarine fiber become significant if our launch power is $50 \mathrm{~mW}(17 \mathrm{dBm})$, so we can assume SPM will be insignificant for the proposed system because the maximum launched power will not exceed $1 \mathrm{~mW}(0 \mathrm{dBm})$.

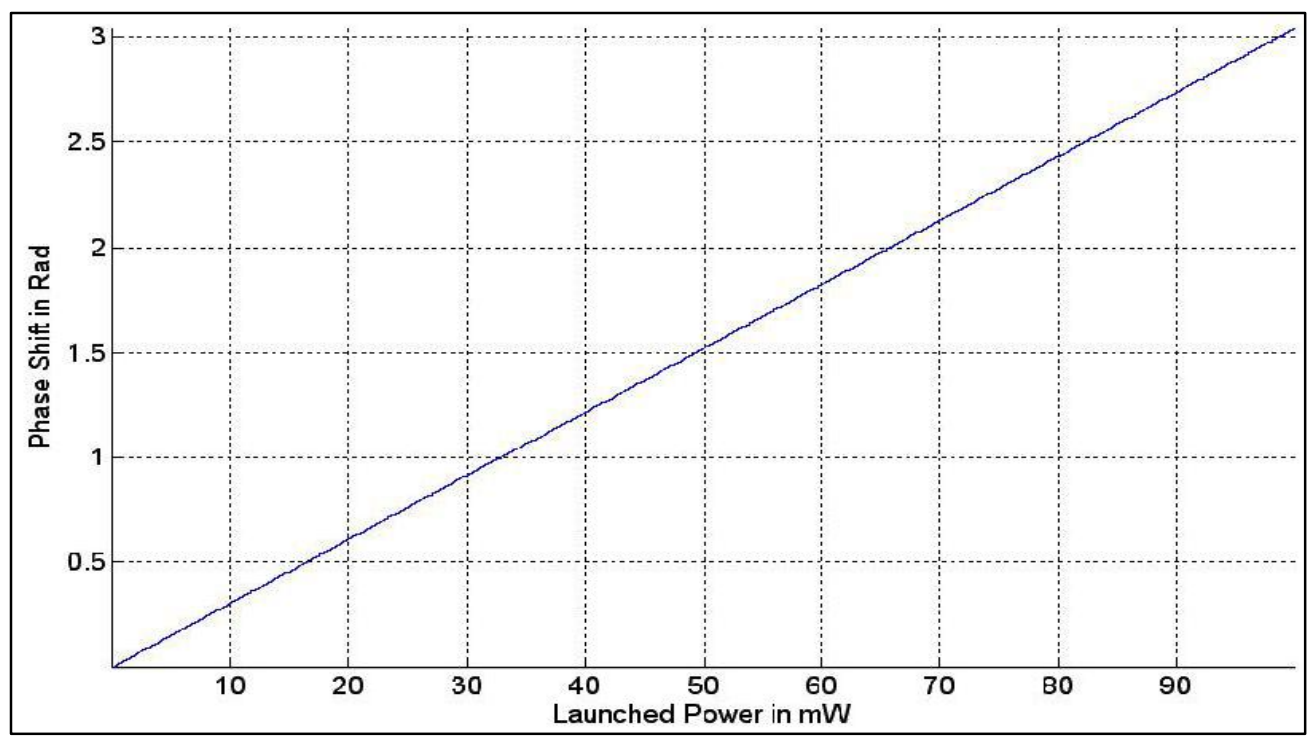

Figure (5): SPM of CorningLEAF_submarine fiber

\section{4 - Fiber Optics System}

Basically, in a fiber optic system, information (such as voice, data or video) is transmitted over fiber in the following way: once encoded into electrical signals, these get converted into light signals that travel down the fiber until they reach a "detector" which then changes the light signals back into electrical signals. Finally, the electrical signals are decoded into information in the form of voice, data, or video [12,13]. OPTSIM 3.6 packet software was used to simulate the optical gigabit Ethernet (transmitter and receiver). The Architecture of 
transmitter and receiver fiber optic system is shown in figure (6). This figure shows the optical transmitter in one node connected with the optical receiver in another node. While figure (7) show the transmitter and receiver connected between two nodes.

The main components of a fiber optic system include:

1) Transmitter. A transmitter converts information - such as voice, data, or video, encoded into electrical signals to light signals. The transmitter receives a modulated electrical signal and converts it into a modulated light signal, after which it sends the light signal into the fiber optic cable. A Light Emitting Diode (LED) or a Laser can be used for generating the light signals.

2) Fiber optic cable - the medium which carries the signal.

3) Receiver. At the other end of the fiber optic cable from the transmitter is the receiver which uses a photo detector to convert the incoming light signal back into an electrical signal. The wavelength designation of the receiver must match that of the transmitter. Important characteristics of receivers are System Performance, which is the Bit Error Rate (BER) for digital systems or Signal to Noise Ratio (SNR) for analog, Saturation and Sensitivity [14]. This accepts the light signal and converts it back into a modulated electrical signal. Table (2) shows the Characteristic of Optical System Design.

\section{5 - Simulation and Analysis Results}

At the beginning, the place of the center states was explored, and the distance between these states was calculated using the google earth software. Moreover, to be on the safe side, a $50 \mathrm{~km}$ distance was added to each distance between the centers of states. Then created three main star topology, the first to connect the north states groups, the second connect the middle states groups, and the last to connect the south states groups. Figure (8) shows the distributed three network groups as a star - bus topology. One city in each main star topology selected as a center depended on the short distances between the cities. Also to get the maximum fiber length not greater than 250km, cities (5- KIRKUK),(1- BAGHDAD) and (11- NASIRIYA) were selected as main centers for the north, middle, and south topology groups respectively, and in these cities the optical switch layer three is used to connect the optical switches layer two in another cities. Because the non-linear effectives (SBS), the fiber length limited to $(250 \mathrm{~km})$, and the maximum allowed transmitted power is $(1 \mathrm{~mW})$ as shown in figure (3). So switch in the city (7- TIKRIT) used to connect between the center of the north and center of the middle network groups, and for the same reason the switch in city (10-DIWANIYA) used to connect between the center of the middle and the center of the south network groups. The reason for selecting the KIRKUK city as center in the north network topology, is the maximum distances between KIRKUK and DOHUK cities is $250 \mathrm{~km}$ as shown in table (3), and this is the maximum distance allowed to prevent the effects of SRS and SBS. On the other hand, the distance between KIRKUK -center of north network topology- and BAGHDAD center of middle network topology- is $(290 \mathrm{~km})$ it is greater than allowed distance $(250 \mathrm{~km})$, for this reason we select TIKRIT city to connect between the north and middle network groups, while the distance between TIKRIT and BAGHDAD is (200km) as shown in table (4). To the same reason, we select DIWANIYA city to connect between center of the middle and center of the south network groups. The distance between the BAGHDAD -center of middle network topology- and NASIRIYA - center of south network topology is $(360 \mathrm{~km})$. While the distance between DIWANIYA and BAGHDAD is (210km) as shown in table (4). 


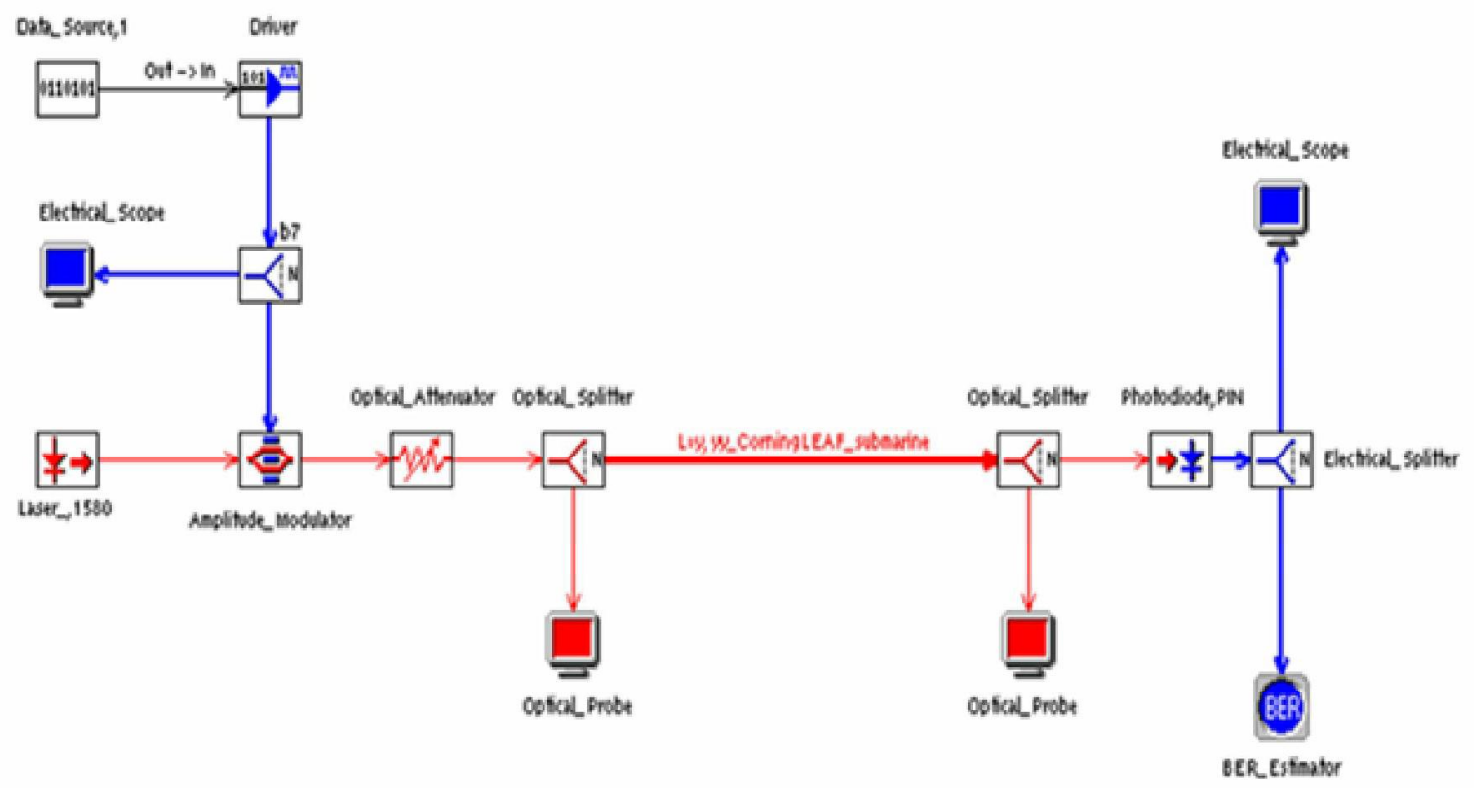

Figure (6): The Architecture of transmitter and receiver fiber optic system

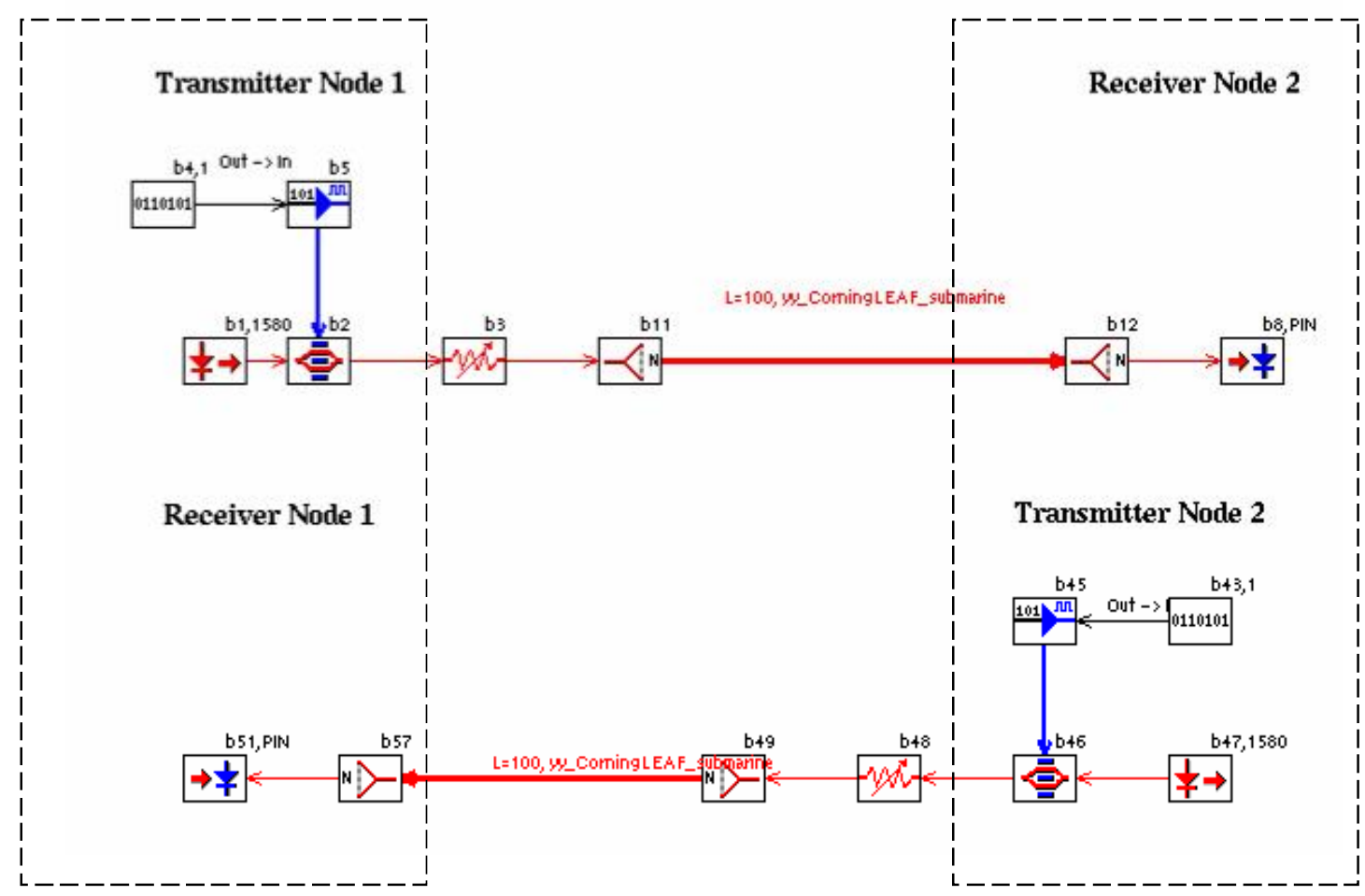

Figure (7): The Architecture of Two Nodes Fiber Optic System 
Table (2): Characteristic of Optical System Design

\begin{tabular}{|r|r|}
\hline BIT RATE & 1 GBITS/SECOND \\
\hline Number of Channels & 1 channel \\
\hline Channel Wavelength & $1580 \mathrm{~nm}$ \\
\hline Bit Error Rate & $10^{-10}$ \\
\hline Digital SNR (Q) & 7 \\
\hline Maximum, fiber length & $250 \mathrm{~km}$ \\
\hline Minimum fiber Length & $\mathrm{km} 100$ \\
\hline Maximum transmitted power & $0 \mathrm{dBm}$ \\
\hline Maximum Attenuation & $58.4 \mathrm{~dB}$ \\
\hline Minimum Attenuation & $18.6 \mathrm{~dB}$ \\
\hline Maximum Launched power & $0 \mathrm{~dB}$ \\
\hline Receiver Quantum Efficiency & $10 \mathrm{Mhz}$ \\
\hline Transmitter Laser Line Width (FWHM) & 0.7 \\
\hline Receiver Responsivity (at reference frequency) & $0.892041905713 \mathrm{~A} / \mathrm{W}$ \\
\hline Channel Coding & $2 \mathrm{GHZ}$ \\
\hline Receiver Single-Pole Electrical Filtering \\
$(-3 \mathrm{~dB}$ Bandwidth) & $0.1 \mathrm{nA}$ \\
\hline Receiver Dark Current & $1580 \mathrm{~nm}$ \\
\hline Feceiver Reference Wavelength & $0.2 \mathrm{~dB}$ \\
\hline Fiber attenuation & $0 \mathrm{ps} / \mathrm{nm} / \mathrm{km}$ \\
\hline Fiber Chromatic Dispersion Slop at 1580 & $\mathrm{m}{ }^{2} \mu 71$ \\
\hline Fiber Mode Field Diameter & $<0.1 \mathrm{ps} / \mathrm{km}$ \\
\hline
\end{tabular}

Table (3): Fiber length and quantity power transmitted to get the BER equal

\begin{tabular}{||c|c|c|c|c|c||}
\hline CITY & $\begin{array}{c}\text { DOHU } \\
\text { K }\end{array}$ & $\begin{array}{c}\text { MOSU } \\
\text { L }\end{array}$ & ERBIL & $\begin{array}{c}\text { SULAIMAN } \\
\text { IYA }\end{array}$ & $\begin{array}{c}\text { TIKRI } \\
\text { T }\end{array}$ \\
\hline $\begin{array}{c}\text { Real distance } \\
\text { to KIRKUK }\end{array}$ & $\begin{array}{c}200 \\
\mathrm{~km}\end{array}$ & $149 \mathrm{~km}$ & $87 \mathrm{~km}$ & $97 \mathrm{~km}$ & $115 \mathrm{~km}$ \\
\hline $\begin{array}{c}\text { Approximate } \\
\text { distance to } \\
\text { KIRKUK }\end{array}$ & $\begin{array}{c}250 \\
\mathrm{~km}\end{array}$ & $200 \mathrm{~km}$ & $140 \mathrm{~km}$ & $150 \mathrm{~km}$ & $170 \mathrm{~km}$ \\
\hline $\begin{array}{c}\text { Power } \\
\text { transmitter }\end{array}$ & $\mathbf{0 d B m}$ & $-10 \mathrm{dBm}$ & $\begin{array}{c}- \\
21.67 \mathrm{dBm}\end{array}$ & $-20 \mathrm{dBm}$ & $\begin{array}{c}-16 \\
\mathrm{dBm}\end{array}$ \\
\hline
\end{tabular}


Alabady: Design and Simulation of an Optical Gigabit Ethernet Network

Table (4): Fiber length and quantity power transmitted required to get the BER equal (1e-10) at $1 \mathrm{Gbps}$, and $1580 \mathrm{~nm}$ wavelength, in middle network groups

\begin{tabular}{|c|c|c|c|c|c|c|c|c|}
\hline$\overline{\text { CITY }}$ & $\begin{array}{c}\text { TIKR } \\
\text { IT }\end{array}$ & $\begin{array}{c}\text { BAQU } \\
\text { BA }\end{array}$ & RAMADI & $\begin{array}{c}\text { KARBAL } \\
\text { A }\end{array}$ & $\begin{array}{c}\text { HILL } \\
\text { A }\end{array}$ & $\overline{\text { KUT }}$ & $\overline{\text { NAJAF }}$ & $\begin{array}{c}\text { DIWANIY } \\
\text { A }\end{array}$ \\
\hline $\begin{array}{c}\text { Real } \\
\text { distance to } \\
\text { BAGHDA } \\
\text { D }\end{array}$ & $\begin{array}{l}150 \\
\text { km }\end{array}$ & $50 \mathrm{~km}$ & $104 \mathrm{~km}$ & $89 \mathrm{~km}$ & $93 \mathrm{~km}$ & $160 \mathrm{~km}$ & $146 \mathrm{~km}$ & $157 \mathrm{~km}$ \\
\hline $\begin{array}{c}\text { Approxima } \\
\text { te distance } \\
\text { to } \\
\text { BAGHDA } \\
\text { D }\end{array}$ & $\begin{array}{l}200 \\
\text { km }\end{array}$ & $100 \mathrm{~km}$ & $155 \mathrm{~km}$ & $140 \mathrm{~km}$ & $\begin{array}{l}150 \\
\text { km }\end{array}$ & $210 \mathrm{~km}$ & $200 \mathrm{~km}$ & $210 \mathrm{~km}$ \\
\hline $\begin{array}{c}\text { Power } \\
\text { transmitter }\end{array}$ & $\begin{array}{c}- \\
10 \mathrm{~dB} \\
\mathrm{~m}\end{array}$ & -30dBm & $\begin{array}{c}-\overline{18.86 \mathrm{dBm}} \\
\end{array}$ & $-22 \mathrm{dBm}$ & $\overline{-}$ & $-8.24 \mathrm{dBm}$ & $-10 \mathrm{dBm}$ & $-8.24 \mathrm{dBm}$ \\
\hline
\end{tabular}

Table (5): Fiber length and quantity power transmitted to get the BER equal (1e-10) at 1Gbps, and 1580nm wavelength, in south network groups

\begin{tabular}{||c|c|c|c|c||}
\hline CITY & $\begin{array}{c}\text { DIWANIY } \\
\text { A }\end{array}$ & SAMAWA & AMARA & BASARH \\
\hline $\begin{array}{c}\text { Real } \\
\text { distance to } \\
\text { NASIRIYA }\end{array}$ & $160 \mathrm{~km}$ & $100 \mathrm{~km}$ & $125 \mathrm{~km}$ & $160 \mathrm{~km}$ \\
\hline $\begin{array}{c}\text { Approxima } \\
\text { te distance } \\
\text { to } \\
\text { NASIRIYA }\end{array}$ & $210 \mathrm{~km}$ & $150 \mathrm{~km}$ & $175 \mathrm{~km}$ & $210 \mathrm{~km}$ \\
\hline $\begin{array}{c}\text { Power } \\
\text { transmitter }\end{array}$ & $-8.24 \mathrm{dBm}$ & $-20 \mathrm{dBm}$ & $-15.23 \mathrm{dBm}$ & $-8324 \mathrm{dBm}$ \\
\hline \hline
\end{tabular}

In the figure (8), there are tow type of switches the first (green and blue color) is layer 2 switch that offer a frame forwarding service based on the physical addresses that are available as part of Layer 2 (i.e., the MAC address of the destination) as well as performing the signal regeneration functions of a repeater. this type of switch will configure to work with multi VLAN. The second type is switch layer 3 (red color) this type of switch work as router that offer a packet forwarding service based on the logical destination addresses (IP address) that are available as part of Layer 3 (as well as providing bridging and repeating functions). This type of switch use Layer 3 header information (packet header) to make selective forwarding decisions, allowing broadcast and multicast traffic to be suppressed. Multiple active paths between communicating hosts can be supported. Conversions between different types and speeds of data link (such as LAN-WAN conversions) are supported by routers. Switch layer 3 are also intelligent enough to provide functions such as access controls based on the type of application protocol being carried and various forms of packet filtering. The reality is that a Layer 3 Switch is simply a class of high performance router that is optimized for the campus LAN or intranet, incorporate routing functionality which allows the switch to perform inter VLAN routing, used to connect between two networks at the network layer. 
The defining characteristics of these devices is their ability to work with switched LANs, to support Gigabit Ethernet speeds, and to handle more complex traffic patterns efficiently, and this is the one of goals for using this type of switch in this proposed network system.

Each layer 2 switch in the state center will be configure to work with multi VLAN as static VLAN to connect different offices in the state, on the other hand the layer 3 switch (router) used to connect between the networks (north, middle and south), inter VLANs and filtering the packets.

From the simulation results, figures $(9,10)$ show the optical spectrum signal at the transmitter and receiver respectively at $1 \mathrm{Gbps}$ to achieve BER (1e-10) at wavelength $1580 \mathrm{~nm}$. These figures clearly show the maximum power transmitter and receiver at $1580 \mathrm{~nm}$ wavelength. In addition, the transmitted power is leases than $(0 \mathrm{dBm})$ therefore the SRS and SBS effects not find on the system. Figure (11) shows the fiber cable length versus power transmitted at bit rate $1 \mathrm{Gbps}, 1580 \mathrm{~nm}$ wavelength to achieve BER (1e-10), the figure show we must increase the transmitted power when the fiber optic length increase, but also we must taking into account the maximum transmitted power not pass $(1 \mathrm{~mW})$ to prevent the effects of SBS and SRS in this system. Figure (12) shows the Eye diagram at the receiver, this figure gives indicator for the systems performance.

\section{6 - Conclusion}

This paper demonstrates the design and simulation of optical gigabit Ethernet, to connect between the states in IRAQ as case study, using optical system software (OPTSIM 3.6).

The results obtained from the proposed design indicate that one can prevent or minimize the linear effects including attenuation and dispersion, and non-linear effects including Stimulated Brillonin Scattering (SBS), Stimulated Raman Scattering (SRS), four wave mixing (FWM), self-phase modulation (SPM), cross-phase modulation (XPM). In addition, one can get the best performance when using longer wavelength (L-band) $1580 \mathrm{~nm}$, the effect of attenuation and dispersion in this wavelength decrease to the minimum. The effect of (SBS), (SRS) and (SPM) will be insignificant by using lower power transmitter $0 \mathrm{dBm}$.

\section{References}

[1]: Chinlon Lin, "Broadband Optical Access Networks and Fiber-to-the-Home Systems technologies and Deployment Strategies ", John Wiley \& Sons Ltd, 2006

[2]: Vivek Alwayn, " Optical Network Design and Implementation", Cisco Press, 2004.

[3]: Mohammad Ilyas, Hussein T. Mouftah, "The handbook of optical communication networks", CRC Press LLC, 2003

[4]: Govind P. Agrawal, "Nonlinear Fiber Optics", Third Edition, Academic Press, 2001

[5]: Eric J. Mitchell, " Simulation of an Optical Network System for a Space Based High Performance Computer System ",Submitted to the Department of Electrical Engineering and Computer Science in Partial Fulfillment of the requirements for the Degree of Master of Engineering in Electrical Engineering and Computer Science at the Massachusetts Institute of Technology May 22, 2002

[6]: Thomas Sims, "Designing Optical Transmission Networks Principles and Approaches", 16thMarch 2006.

(Available at http://www.webarchive.ja.net/services/events/calendar/2006/opticalnetworking/Verizon.pdf ) 
[7]:E.G. Shapiro, M.P.Fedoruk, and S.K.Turitsyn," Numerical estimate of BER in optical systems with strong patterning effects", Electronics letters. Vol. 37,No. 19,pp. 11791181.2001.

[8]: G.Bosco, A.carena, V.curri, R.Gandino, P.poggiolini, S.Benedetto.”A Novel Analytical Method for the BER Evaluation in Optical Systems Affected by Parametric Gainn", IEEE Photonics Technology letters,Vol. 12,No. 2,February 2000.

[9]: Corning Incorporated, "Single-Mode Dispersion Measurement Method ",ISO 9001 Registered, Supersedes: August 2000, Issued: September 2001.

[10]: Tom Baldwin, Steven Durand, "IF Fiber Selection Criteria" EVLA Memorandum No. 32 Project, National Radio Astronomy Observatory, Washington, D.C., November, 2001.( Available at http://www.aoc.nrao.edu/evla/geninfo/memoseries/evlamemo32.pdf)

[11]: Agrawal, G. P., "Fiber-Optic Communication Systems", John Wiley \& Sons, New York, 1997.

[12]: Yongyut , Prof. P. L. Chu, "Simple Star Multihop Optical Network", IEEE Journal of Lightwave Technology, Vol.19, No.4, pp. 425-432, April 2001.

[13]: Yongyut , Prof. P. L. Chu, "A New Multihop Optical Network: Simple Star", Optical Network Magazine, Vol. 3, Issue 4, July/August 2002.

[14]: P. Green, "Progress in optical networking", IEEE Communications Magazine, Vol. 39, pp. 54-61, Jan.2001.

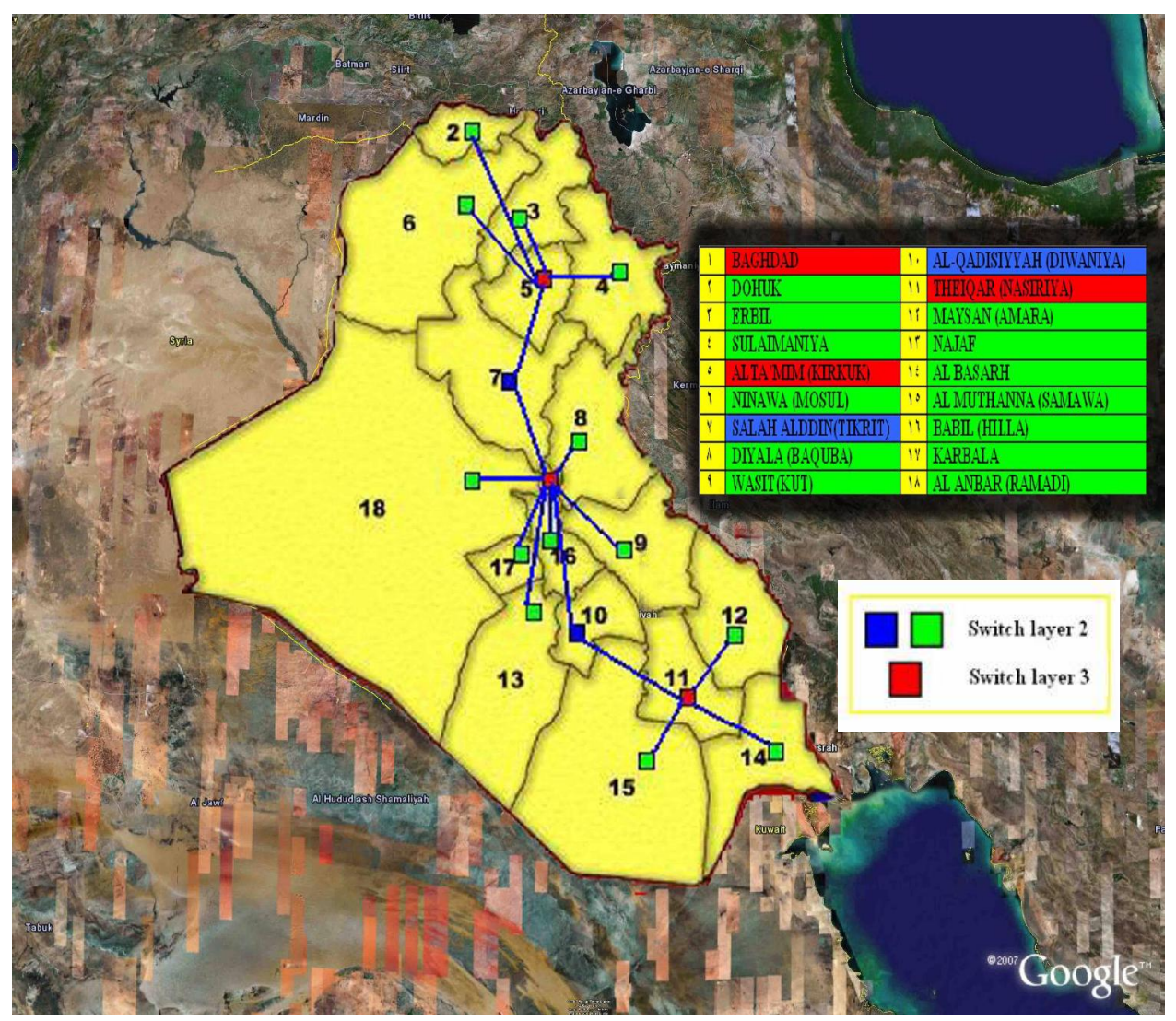

Figure (8): The distributed three networks groups as a star - bus topology 


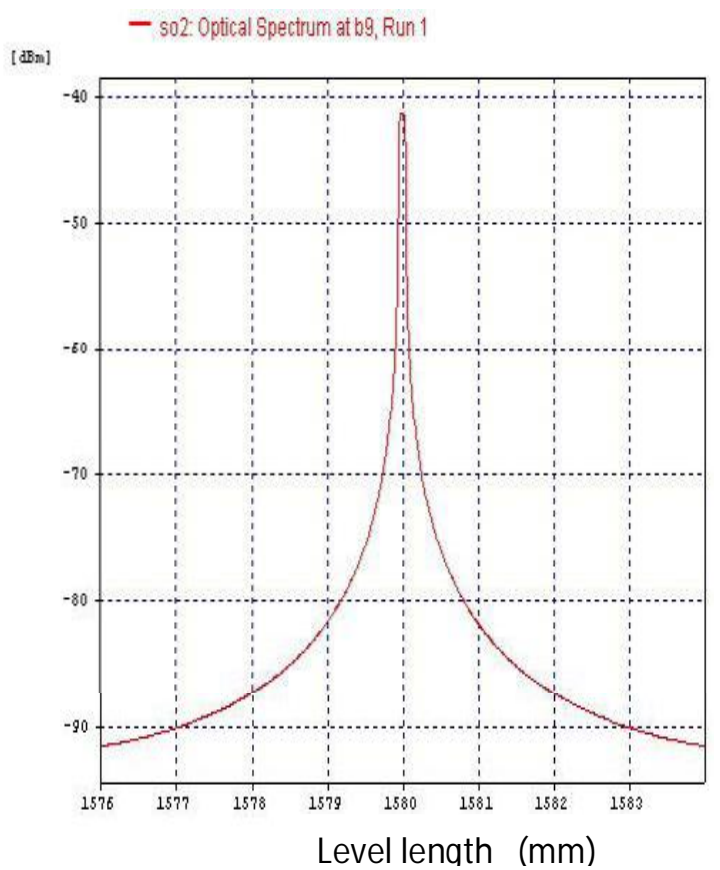

Figure (9) : Optical power spectrum at the transmii

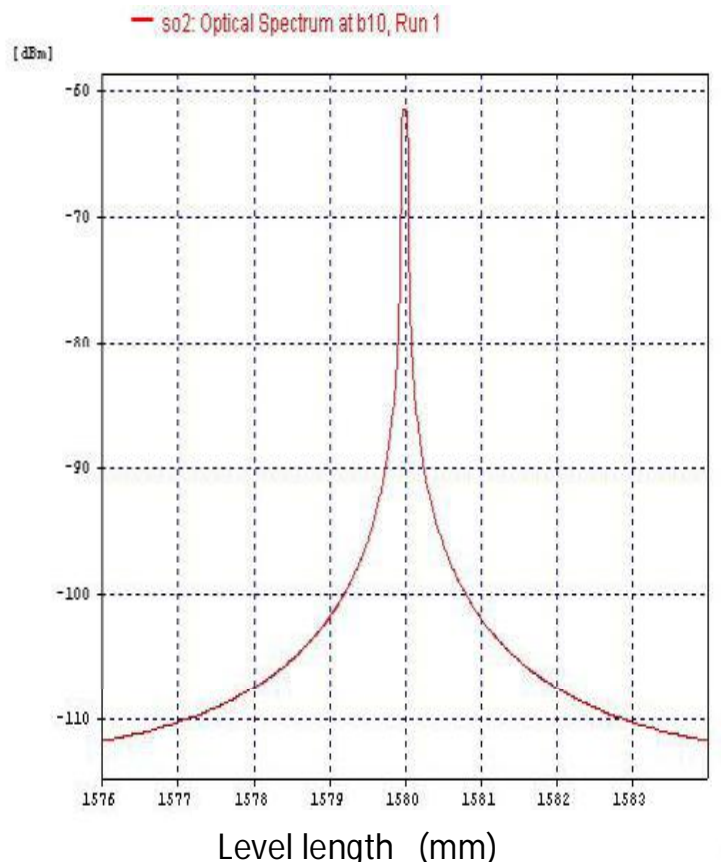

Figure (10) : Optical power spectrum at the

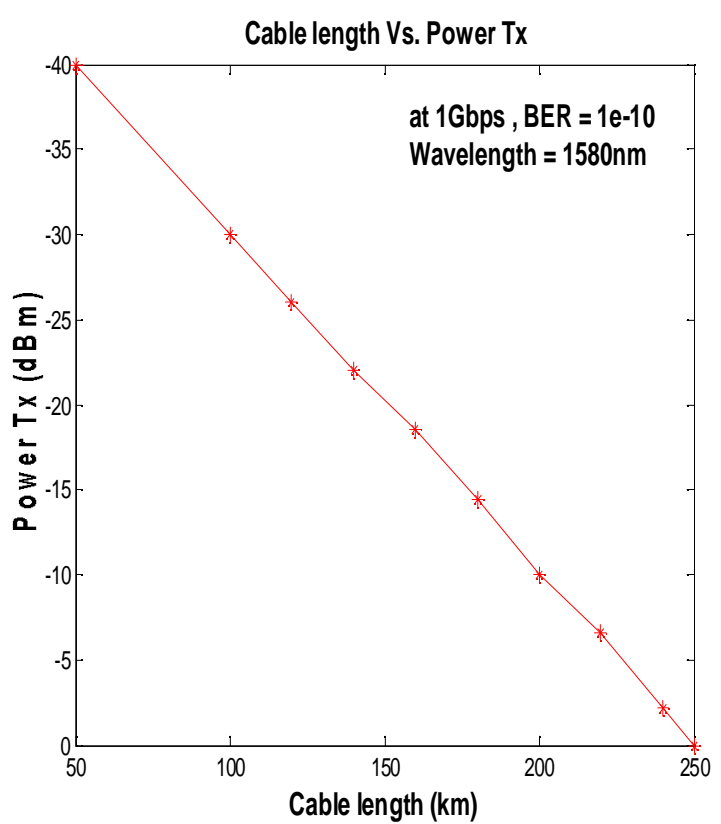

Figure (11): Cable length Vs. power transmitted at 1Gbps,

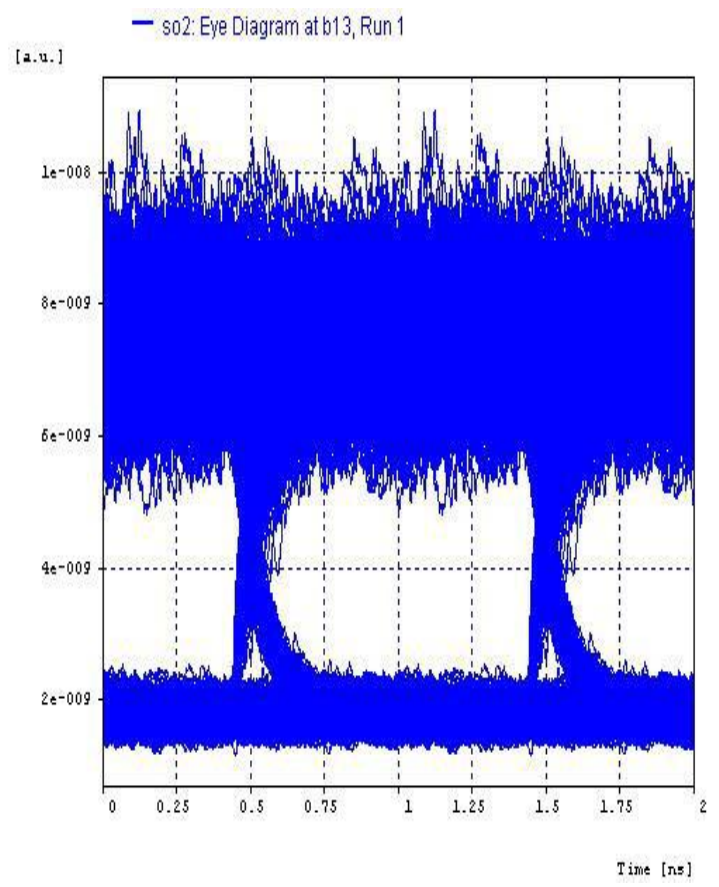

Fiaure (12): Eve diaaram at the

The work was carried out at the college of Engg. University of M osul 